\title{
Mashiningan Improves Opioid-Induced Constipation in Rats by Activating Cystic Fibrosis Transmembrane Conductance Regulator Chloride Channel
}

\author{
Yumi Harada, Seiichi lizuka, Yayoi Saegusa, Sachiko Mogami, Naoki Fujitsuka, \\ and Tomohisa Hattori
}

Tsumura Kampo Research Laboratories, Tsumura \& Co., Ibaraki, Japan (Y.H., S.I., Y.S., S.M., N.F., T.H.)

Received February 8, 2017; accepted April 21, 2017

\begin{abstract}
Opioid receptor stimulants are analgesics used in patients with and without cancer; however, they often cause constipation, resulting in poor adherence and deterioration of the quality of life. Hence, suitable treatments for constipation are required. In this study, we investigated the pharmacological mechanisms of action of mashiningan (MNG), a Kampo medicine used to treat constipation, and evaluated the effect of MNG on opioid-induced constipation in rats. MNG (100 or $300 \mathrm{mg} / \mathrm{kg}$ ) was orally administered to normal or codeine phosphate $(\mathrm{CPH})$-induced constipation in rats, and its effect was evaluated on the basis of fecal counts, characteristics, and weight. Small intestinal fluid secretion was measured after treatment with MNG alone or coadministration with a cystic fibrosis transmembrane conductance regulator (CFTR)-specific inhibitor (CFTRinh-172). The effects of MNG on the CFTR and type-2
\end{abstract}

chloride channel were determined using patch-clamp or shortcircuit current experiments, respectively. MNG increased the fecal weight and proportion of soft feces in normal rats. $\mathrm{CPH}$-induced constipation in rats decreased fecal counts and weight, whereas MNG prevented these effects and increased the proportion of soft feces. MNG increased the electronic chloride current, and this effect was inhibited by the CFTRinh-172 in the CFTR assay. Furthermore, MNG increased small intestinal fluid secretion, and this effect was abolished by coadministration with the CFTRinh172. MNG improved opioid-induced constipation in rats, and this improvement may have been mediated by increasing intestinal fluid secretion via CFTR chloride channel activation. Therefore, MNG is expected as a medicine of the treatment of constipation in patients taking opioids.

\section{Introduction}

Constipation is a common disorder of the digestive tract observed in 12-19\% of people (Gras-Miralles and Cremonini, 2013), especially in the elderly aged 65 years or older in Asia (Kurniawan and Simadibrata, 2011). The number of patients with cancer is currently increasing gradually, and these patients need pain relief at all stages of their disease. The mainstay of the management of cancer pain is opioid-based pharmacotherapy (Canadian Agency for Drugs and Technologies in Health, 2014). However, the use of opioids in the treatment of chronic pain in patients with and without cancer causes bowel disorders as an adverse effect. The most frequent of these disorders is constipation, which often results in the discontinuation of opioid therapy (McNicol et al., 2003; Gyawali et al., 2015; Sani and Mahan, 2015; Nelson and Camilleri, 2016). Therefore, opioid-induced constipation compromises pain management.

For severe constipation, proactive medical treatment is required to improve the quality of life of patients and increase their satisfaction with therapy (LoCasale et al., 2016).

Y.H., S.I., Y.S., S.M., N.F., and T.H. are employed by Tsumura \& Co. https://doi.org/10.1124/jpet.117.240630.
Currently, various medications are available for treating constipation, such as laxatives and enemas, depending on the symptom and type (Prichard et al., 2016). Additionally, chloride channel activators have drawn attention as new medicines that are associated with intestinal fluid secretion. It has been reported that phenylquinoxalinone, a cystic fibrosis transmembrane conductance regulator (CFTR) activator, normalizes stool output in a mouse model of opioidinduced constipation (Cil et al., 2016, 2017). Another agent, lubiprostone, increases osmotic pressure in the intestinal gut lumen by transporting chloride ions and promoting the secretion of intestinal fluid (Jakab et al., 2012), although the mechanism of lubiprostone-induced type- 2 chloride channel (ClC-2)-mediated chloride secretion remains controversial. Linaclotide, a guanylate cyclase-C (GC-C) receptor agonist, activates CFTR chloride channel, leading to the transport of chloride ions $\left(\mathrm{Cl}^{-}\right)$, bicarbonate ions $\left(\mathrm{HCO}_{3}^{-}\right.$), and water to the intestinal lumen (Vaandrager et al., 1998; Sindic and Schlatter, 2006; Bryant et al., 2010). It improved constipation by softening the stool by increasing its water content, which promotes intestinal transportation (Busby et al., 2010; Sharma et al., 2013; Yu and Rao, 2014). However, the use of these medicines, including laxatives, requires caution because of possible side effects,

ABBREVIATIONS: CFTR, cystic fibrosis transmembrane conductance regulator; CFTRinh-172, CFTR-specific inhibitor; ClC-2, type-2 chloride channel; $\mathrm{CPH}$, codeine phosphate; DW, distilled water; GC-C, guanylate cyclase-C; IP, intraperitoneal; MNG, mashiningan; NhBE, normal human bronchial epithelial cell; $\mathrm{OP}_{3}$, opioid $\mu$; PBS, phosphate-buffered saline containing $0.3 \%$ dimethyl sulfoxide; PO, per os. 
such as habit formation, abdominal pain, and nausea (Izzy et al., 2016).

Mashiningan (MNG), a Kampo medicine composed of six crude drugs, is widely prescribed to patients with abdominal sensations such as lumpy or hard stools and symptoms of frailty after illness or associated with aging in Japan and other Asian countries (Iizuka and Hamamoto, 2015; Nakae et al., 2016). The efficacy and safety of MNG in alleviating functional constipation symptoms have also been reported in randomized double-blind, placebo-controlled studies in China (Cheng et al., 2011; Zhong et al., 2013). However, its precise mechanism of action has not been fully understood. Therefore, in the present study, we investigated the pharmacological mechanisms of action of MNG and evaluated the effect of MNG on opioid-induced constipation in rats.

\section{Materials and Methods}

\section{Animals}

Six-week-old male Sprague-Dawley rats (weight, 160-210 g) were purchased from Japan Charles River (Kanagawa, Japan). All the animals were housed in stainless steel cages in a room with controlled ambient temperature $\left(23 \pm 3^{\circ} \mathrm{C}\right)$, humidity $(50 \pm 20 \%)$, and lighting (12-hour light:dark cycle) conditions. The animals were provided water and a standard laboratory animal diet (MF; Oriental Yeast, Tokyo, Japan) ad libitum. Experiments were performed using free-fed rats, except where otherwise mentioned. All experimental procedures were performed according to the Guidelines for the Care and Use of Laboratory Animals and approved by the Laboratory Animal Committee (Permits 15-021, 15-036, 16-044, and 16-053) of Tsumura (Tokyo, Japan).

\section{Drugs and Treatments}

Codeine phosphate $(\mathrm{CPH})$ was purchased from Takeda Pharmaceutical (Osaka, Japan). MNG was supplied by Tsumura in the form of a powdered extract obtained by spray-drying a hot water extract mixture of the following six crude drugs: Cannabis Fructus $(5.0 \mathrm{~g})$, Armeniacae Semen (2.0 g), Paeoniae Radix (2.0 g), Rhei Rhizoma (4.0 g), Magnoliae Cortex (2.0 g), and Aurantii Fructus Immaturus $(2.0 \mathrm{~g})$. CPH and MNG were dissolved in distilled water (DW) prior to oral [per os (PO)] administration. CFTR-specific inhibitor (CFTRinh-172; Tocris, Bristol, UK) was dissolved in phosphatebuffered saline containing $0.3 \%$ dimethyl sulfoxide (PBS) prior to intraperitoneal (IP) administration. The other analytical reagents used, including the commercially available products, were of the highest purity. Treatments were administered to unanesthetized and lightly hand-restrained rats at $5 \mathrm{~mL} / \mathrm{kg}$ (PO) or $1 \mathrm{~mL} / \mathrm{kg}$ (IP) doses.

\section{Experimental Protocols}

Effect of MNG on Defecation in Rats. MNG (100 or $300 \mathrm{mg} / \mathrm{kg}$ ) or DW was orally administered to normal rats 2 hours before the dark cycle commenced, and the fecal count was evaluated 16 hours after administration. We chose MNG doses (100 and $300 \mathrm{mg} / \mathrm{kg}$ ) that did not influence the food intake of the free-fed rats in this experiment.

The feces were dried for more than 1 day at approximately $45^{\circ} \mathrm{C}$, the weight was measured, and the weight per count was calculated. The fecal characteristics were macroscopically assessed based on criteria modified in a previous report and described as follows (Bhol and Schechter, 2007): normal feces: well-formed pellets, rigid as normal; soft feces: formed pellet with moisture, soft feces that retains its shape; and diarrhea: loose feces with abnormal form, much moisture, and softer shapeless form.
Effect of MNG on Opioid-Induced Constipation in Rats. Rats were administered (PO) the vehicle or CPH (12 mg/kg) 2.5 hours before the dark cycle onset. MNG (100 or $300 \mathrm{mg} / \mathrm{kg}$ ) or DW was then administered (PO) 0.5 hour after the $\mathrm{CPH}$ treatment. The feces were collected for 16 hours and counted, and then the characteristics were assessed. After drying for more than 1 day at approximately $45^{\circ} \mathrm{C}$, the fecal weight was measured.

Measurement of Small Intestinal Fluid Secretion. The effect of MNG on the small intestinal fluid secretion in rats was measured. DW or MNG $(100,300$, and $1000 \mathrm{mg} / \mathrm{kg}$ ) was administered to 24 -hour fasted normal rats, and, 1 hour later, the rats were anesthetized with isoflurane to collect intestinal fluid. The initial portion of the duodenum (pylorus) and cecum was clamped using forceps and excised, and then the intestinal fluid content between the duodenum and cecum was measured. In a parallel experiment, 24-hour fasted rats were IP injected with a CFTR-specific inhibitor (CFTRinh-172, $1 \mathrm{mg} / \mathrm{kg}$ ) 30 minutes before PO administration of DW or MNG $(1000 \mathrm{mg} / \mathrm{kg})$, and then the fluid content of the intestine was measured, as described above. The dose of CFTRinh-172 was determined with reference to previous studies (Thiagarajah et al., 2004; Akiba et al., 2005; Mizumori et al., 2009).

Measurement of Chloride Channel Activity. To measure the CFTR chloride channel activity, cryopreserved primary normal human bronchial epithelial cells (NhBE; LifeLine Cell Technology, Frederick, MD) were expanded and transferred to Snapwell inserts to form an epithelium. Cells were plated on the Snapwell permeable inserts and were grown for at least 21 days in differentiation medium exposed to an air-liquid interface from day 4 to promote differentiation. Polarized NhBE were differentiated on a permeable support exposed to an air-liquid interface and transferred to Ussing chambers containing HEPES-buffered physiologic saline, and the transepithelial potential was voltage clamped to $0 \mathrm{mV}$. Apical membrane currents from the epithelial sodium channel were blocked with benzamil (final concentration, $10 \mu \mathrm{mol} / \mathrm{L}$; Sigma-Aldrich, St. Louis, MO). Forskolin (final concentration, $10 \mu \mathrm{mol} / \mathrm{L}$; Sigma-Aldrich) was applied to epithelia to activate CFTR channels. After 30 minutes, when the CFTR peak current had decayed to a plateau level, the vehicle or MNG was cumulatively applied $(50,100,200$, and $400 \mu \mathrm{g} / \mathrm{mL})$ and short-circuit currents $(n=3)$ were measured using a previously reported method with slight modification (Bijvelds et al., 2009; Yang et al., 2011; Cil et al., 2016). CFTRinh-172 (20 $\mu \mathrm{mol} / \mathrm{L}$; Tocris or Sigma-Aldrich) was added at the end to confirm the presence of the CFTR-dependent current.

The effect of MNG on cloned human ClC-2 chloride channels (stably expressed as human CLCN2 in Chinese hamster ovary cells) was evaluated using the automated patch-clamp method (QPatch HT; Sophion Bioscience A/S, Ballerup, Denmark). After applying the vehicle to naive cells using the QPatch robot pipetting system, MNG $\left(400 \mu \mathrm{g} / \mathrm{mL}\right.$ ) or the positive control (cadmium chloride, $\mathrm{CdCl}_{2}$; $100 \mu \mathrm{mol} / \mathrm{L}$; Sigma-Aldrich) was applied at a minimum of 3-minute intervals $(n=3)$.

Binding Affinity for Opioid $\boldsymbol{\mu}$ Receptors. To clarify the involvement of opioid $\mu\left(\mathrm{OP}_{3}\right)$ receptors, the binding affinity was examined. For the radioligand-binding assay of $\mathrm{OP}_{3}$ receptors, $\mathrm{CHO}-\mathrm{K} 1$ cells stably transfected with a plasmid encoding the human $\mathrm{OP}_{3}$ were homogenized in modified Tris-hydrochloride ( $\mathrm{HCl}$ ) buffer, and aliquots were added to MNG $(200 \mu \mathrm{g} / \mathrm{mL})$ and incubated for 60 minutes at $25^{\circ} \mathrm{C}$ with $0.60 \mathrm{nmol}\left[{ }^{3} \mathrm{H}\right]$ diprenorphine. Nonspecific binding was estimated in the presence of $10.0 \mu \mathrm{mol}$ naloxone.

\section{Statistical Analysis}

All values are presented as the mean \pm S.E.M. Statistical analyses of the two groups were performed using the Student's $t$ test. The differences between the mean values of multiple groups were determined using a one-way analysis of variance, followed by the Dunnett or Steel post hoc test. For all tests, statistical significance was set at $P$ values $<0.05$. 


\section{Results}

Effect of MING on Defecation in Normal Rats. As shown in Fig. 1A, the fecal count for 16 hours was $38.7 \pm 1.5(\mathrm{~N})$ in the control group administered DW, which was not significantly different from that of the MNG (100 or $300 \mathrm{mg} / \mathrm{kg}$ )treated group. The fecal weight was $3.8 \pm 0.1 \mathrm{~g}$ in the control group, whereas it was $4.9 \pm 0.2 \mathrm{~g}$ and $5.7 \pm 0.4 \mathrm{~g}$ in the MNG 100 and $300 \mathrm{mg} / \mathrm{kg}$-treated groups, respectively (Fig. 1B). MNG significantly increased the fecal weight of rats in a dosedependent manner. In addition, the results in Fig. $1 \mathrm{C}$ reveal that the fecal weight per count of the MNG-treated group was higher than that of the control group (100 and $300 \mathrm{mg} / \mathrm{kg} \mathrm{MNG}$,
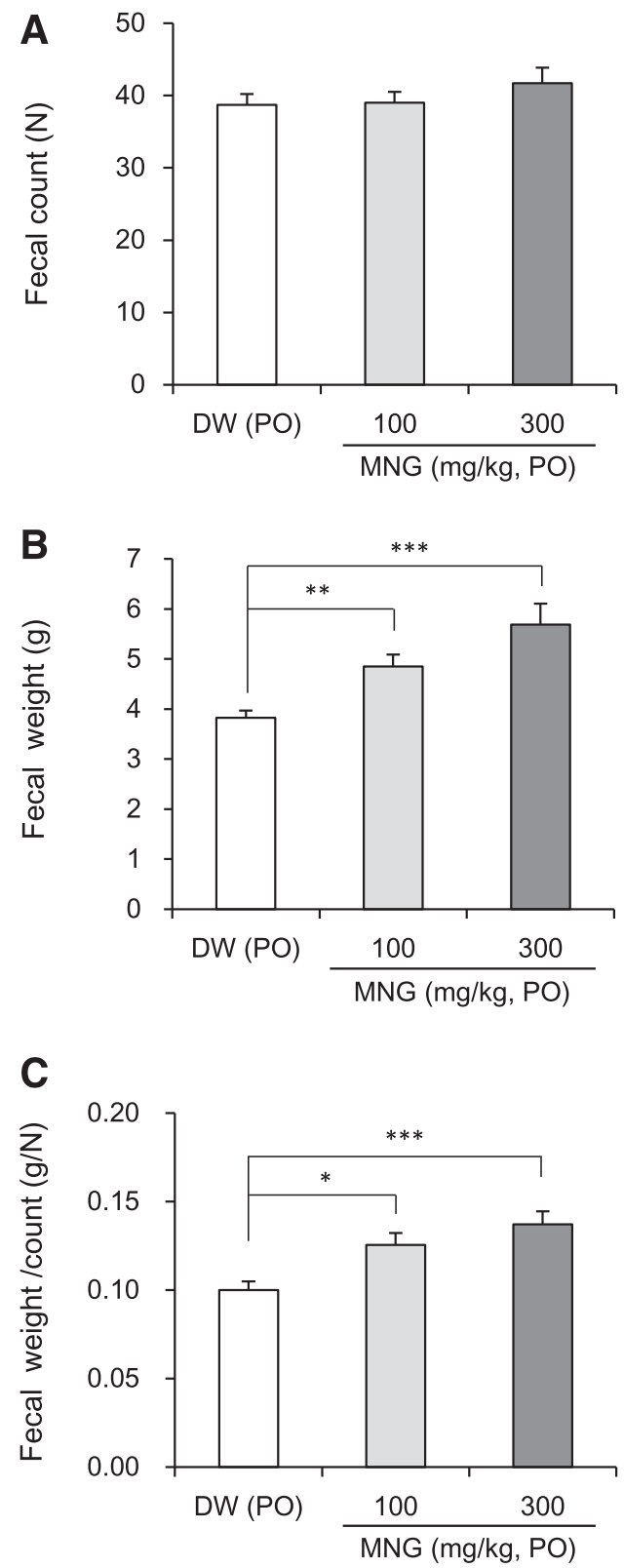

Fig. 1. Effects of MNG on defecation in normal rats. DW or MNG (100 or $300 \mathrm{mg} / \mathrm{kg}$ ) was PO administered to normal rats 2 hours before onset of dark cycle. (A) Fecal count and (B) weight were measured over 16 hours, and $(\mathrm{C})$ weight per fecal count was also calculated. Data are mean \pm S.E.M. $* P<0.05, * * P<0.01$, and $* * * P<0.001$ versus DW-treated normal rats using Dunnett's test or Steel's test $(n=10)$.
$0.13 \pm 0.01$ and $0.14 \pm 0.01 \mathrm{~g}, P<0.05$ and 0.001 , respectively, versus control, $0.10 \pm 0.01 \mathrm{~g}$, Dunnett's test). No difference in food intake was observed between DW- and the MNG (100 or $300 \mathrm{mg} / \mathrm{kg}$ )-treated groups.

The fecal characteristics shown in Table 1 were all normal in the control and MNG $100 \mathrm{mg} / \mathrm{kg}$ groups. In contrast, $17.2 \%$ soft feces were observed in the MNG $300 \mathrm{mg} / \mathrm{kg}$ group $(P<$ 0.01 versus control, Steel's test). Furthermore, diarrhea was not observed in any of the groups.

Effect of MNG on Opioid-Induced Constipation in Rats. The fecal excretion 16 hours after administration of DW or MNG was evaluated, and the typical examples of the feces are shown in Fig. 2. The $\mathrm{CPH}+\mathrm{DW}$-treated group exhibited decreased defecation compared with that of the other groups. Fecal counts were lower in the CPH + DW-treated group (25.6 \pm $1.3, P<0.001$, Steel's test) than in the control group treated with DW alone $(42.3 \pm 2.4)$. The $\mathrm{CPH}+$ MNG-treated group showed significantly increased fecal counts compared with those of the $\mathrm{CPH}+\mathrm{DW}$-treated group (100 and $300 \mathrm{mg} / \mathrm{kg}$ MNG, $34.0 \pm 1.9$ and $34.0 \pm 2.4$, respectively, both $P<0.05$, Steel's test; Fig. 3A).

The fecal weight significantly decreased in the $\mathrm{CPH}+$ DW-treated group $(2.5 \pm 0.1 \mathrm{~g}, P<0.01$, Steel's test $)$ compared with that of the control group $(3.5 \pm 0.2 \mathrm{~g})$, and this effect was recovered by coadministration of $\mathrm{MNG}(100 \mathrm{mg} / \mathrm{kg} \mathrm{MNG}$; $3.0 \pm 0.1 \mathrm{~g}, 300 \mathrm{mg} / \mathrm{kg}$ MNG; $3.1 \pm 0.3 \mathrm{~g}, P<0.05$, Steel's test; Fig. 3B). Food intake did not differ significantly between groups. The evaluation of the fecal characteristics revealed that 5.0\% of the total feces was soft in the CPH + MNG $300 \mathrm{mg} / \mathrm{kg}$ group ( $P<0.01$ versus control, Steel's test), but that of the other groups was normal (Table 2). In addition, no diarrhea was observed in any group.

Effect of MNG on Small Intestinal Fluid Secretion in Normal Rats. The effect of MNG on the small intestinal fluid secretion in 24-hour fasted rats was assessed. As shown in Fig. $4 \mathrm{~A}$, the fluid content of the small intestine 1 hour after administration was $0.62 \pm 0.07 \mathrm{~g}$ in the DW-treated control group. MNG at doses of 300 and $1000 \mathrm{mg} / \mathrm{kg}$ significantly increased the amount of the small intestinal fluid in rats (300 and $1000 \mathrm{mg} / \mathrm{kg}, 1.01 \pm 0.11$ and $1.21 \pm 0.08 \mathrm{~g}, P<0.05$ and $P<0.001$, respectively).

The effect of MNG on the small intestinal fluid was abolished by IP injection of the CFTRinh-172 (DW + PBS group, $0.55 \pm 0.07 \mathrm{~g}$; MNG + PBS group, $1.27 \pm 0.10 \mathrm{~g}, P<$ 0.001; and MNG + CFTRinh-172 group, $0.63 \pm 0.06 \mathrm{~g}, P<$ 0.001 ; Fig. 4B). In DW-treated rats, the injection of CFTRinh172 had no effect on the small intestinal fluid (Fig. 4C).

Effects of MNG on Chloride Channel Activities and Opioid Receptors. To clarify the mechanisms of action of

\section{TABLE 1}

Effects of MNG on fecal characteristics in normal rats.

DW or MNG ( 100 or $300 \mathrm{mg} / \mathrm{kg}$ ) was PO administered to normal rats $2 \mathrm{~h}$ before onset of dark cycle. Fecal characteristics were assessed for $16 \mathrm{~h}$ after administration. Data are the mean of incidence $(\%)$.

\begin{tabular}{lrcc}
\hline \multirow{2}{*}{ Fecal Characteristics } & \multicolumn{3}{c}{ Incidence (\%) } \\
\cline { 2 - 4 } & DW & MNG $(100 \mathrm{mg} / \mathrm{kg})$ & MNG $(300 \mathrm{mg} / \mathrm{kg})$ \\
\hline Normal & 100 & 100 & $83^{* *}$ \\
Soft & 0 & 0 & $17^{* *}$ \\
Diarrhea & 0 & 0 & 0 \\
\hline
\end{tabular}

**P $<0.01$ versus DW-treated normal rats using Steel's test $(n=10)$. 
A $\mathrm{DW}$

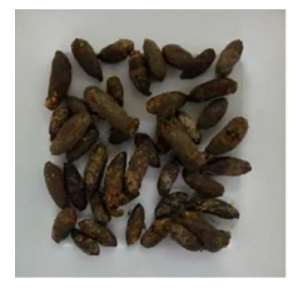

B $\mathrm{CPH}+\mathrm{DW}$

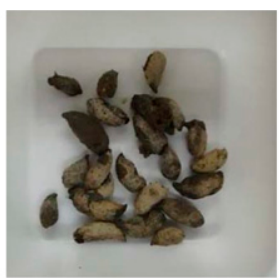

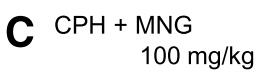

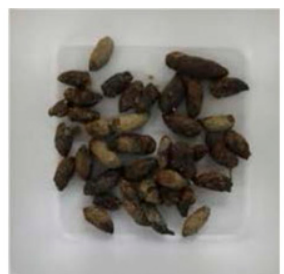

D $\mathrm{CPH}+\mathrm{MNG}$

$300 \mathrm{mg} / \mathrm{kg}$

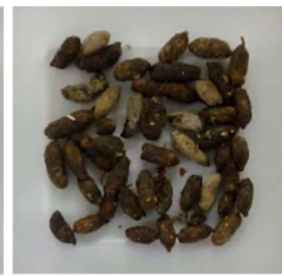

Fig. 2. Representative example of feces in $\mathrm{CPH}-$ induced constipation rat model. (A) DW only, (B) $\mathrm{CPH}+$ $\mathrm{DW}$, (C) $\mathrm{CPH}+\mathrm{MNG} 100 \mathrm{mg} / \mathrm{kg}$, and (D) $\mathrm{CPH}+$ MNG $300 \mathrm{mg} / \mathrm{kg}$; DW or MNG were PO administered 0.5 hour after treatment with (A) DW or (B-D) $\mathrm{CPH}$ $12 \mathrm{mg} / \mathrm{kg}$ (PO). Feces were collected over 16 hours after DW or MNG administration.
MNG, its effects on the CFTR chloride channel activity in NhBE were evaluated using the Ussing chamber assay. The short circuit current was negatively changed in a dosedependent manner by the addition of MNG $(50-400 \mu \mathrm{g} / \mathrm{mL})$ to the cell culture. Treatment with CFTRinh-172 $(20 \mu \mathrm{mol} / \mathrm{L})$, a CFTR-specific inhibitor, blocked the response of MNGtreated cells, which was restored to the levels of the vehicletreated cells (Table 3 ). In contrast, when the activity of the ClC-2 chloride channel was presented as percent inhibition, although an agonist effect is denoted as a negative inhibition value, it was $60.1 \pm 9.4 \%$ in epithelial cells treated with $400 \mu \mathrm{g} / \mathrm{mL}$ MNG.

The effect of $\mathrm{MNG}$ on the $\mathrm{OP}_{3}$ receptor was evaluated using a radioligand-binding assay, and no effect was observed at $200 \mu \mathrm{g} / \mathrm{mL}$ on the binding activities of this receptor (data not shown).

\section{Discussion}

In this study, we found that administration of MNG improved opioid-induced constipation in rats. The effect of MNG was most likely mediated by an increase in the intestinal fluid secretion via CFTR chloride channel activation. First, we clarified the pharmacological effects of MNG on defecation in normal rats, and the results showed that the administration of MNG significantly increased the fecal weight, but not the count. Next, we elucidated the pharmacological effects of MNG on defecation in a CPH-treated rat model of opioid-induced constipation. The decrease in fecal count and weight in the $\mathrm{CPH}$-treated rats was partially prevented by MNG administration.

Increased fecal output (frequency and volume) is one of the important factors in constipation therapy (Chokhavatia et al., 2016). Dry and hard stool causes difficult intestinal transit (Fredericks et al., 2010), and, therefore, it is desirable for feces to be softened by treatment of constipation (Canadian Agency for Drugs and Technologies in Health, 2014; Costilla and Foxx-Orenstein, 2014). In this study, the analysis of fecal characteristics revealed that MNG administration increased the incidence of soft feces in both normal and CPH-treated rats. Our previous study demonstrated that MNG increased the water content and size of feces in rats with fiber-free dietinduced constipation (Harada et al., 2016). Therefore, MNG may have facilitated defecation by softening the feces in both normal and CPH-treated rats. The present study demonstrated that MNG had no binding affinity for the $\mathrm{OP}_{3}$ receptor, suggesting that MNG improved constipation without interfering with the action of opioids.

The enhancement of the small intestinal fluid secretion is important for promoting defecation (De Lisle, 2012; Cil et al., 2016). The increased fluid secretion in the bowel lumen softens the feces and promotes bowel transit (Lacy and Levy, 2007; Chokhavatia et al., 2016). Recently, stimulation of fluid secretion by pharmacologically opening chloride channels in the epithelium of the intestinal mucosa has been considered a new approach in constipation therapy (Schiller, 2004). The selective chloride channel activator lubiprostone enhances the secretion of the small intestinal fluid and promotes the small bowel movement, leading to increased colon transit (Camilleri et al., 2006; Lacy and Levy, 2007). The GC-C receptor agonist linaclotide also increases chloride secretion and thereby

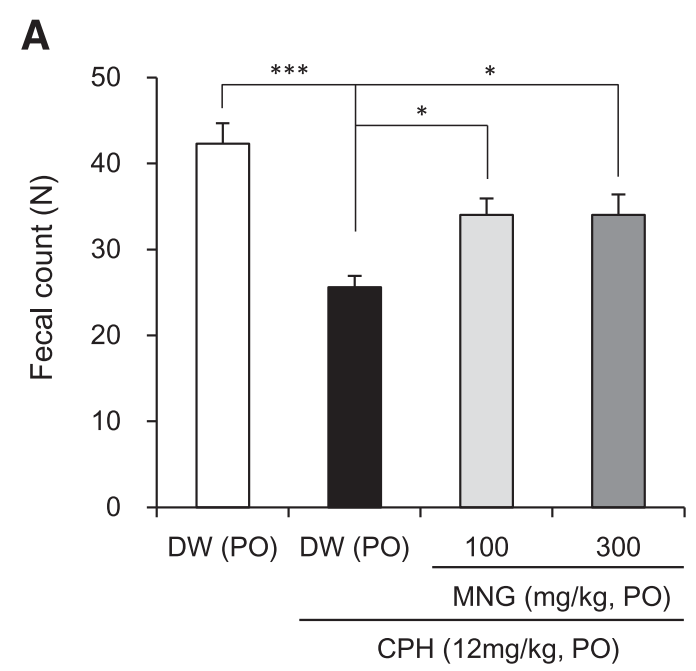

B

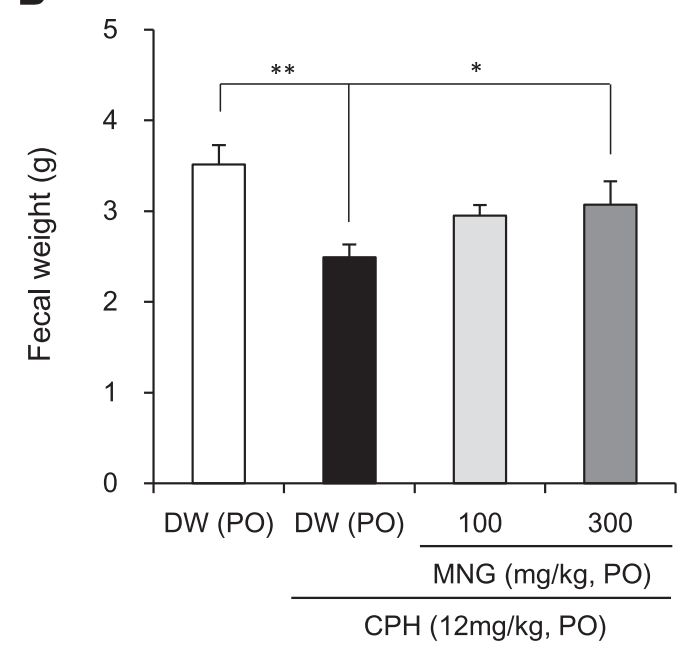

Fig. 3. Effects of MNG on CPH-induced constipation rat model. (A) Fecal count and (B) weight were measured for 16 hours after DW or MNG administration. Data are mean \pm S.E.M. $* P<0.05$, $* * P<0.01$, and $* * * P<0.001$ versus CPH + DW-treated rats using Steel's test $(n=10)$. 
TABLE 2

Effects of MNG on fecal characteristics of CPH-induced constipation rat model.

DW or MNG was PO administered $0.5 \mathrm{~h}$ after DW or CPH $12 \mathrm{mg} / \mathrm{kg}$ treatment. Fecal characteristics were assessed for $16 \mathrm{~h}$ after DW or MNG administration. Data are the mean of incidence (\%).

\begin{tabular}{lcccc}
\hline & \multicolumn{4}{c}{ Incidence (\%) } \\
\cline { 2 - 5 } Fecal Characteristics & Control & $\mathrm{CPH}+\mathrm{DW}$ & $\mathrm{CPH}+\mathrm{MNG}(100 \mathrm{mg} / \mathrm{kg})$ & $\mathrm{CPH}+\mathrm{MNG}(300 \mathrm{mg} / \mathrm{kg})$ \\
\hline Normal & 100 & 100 & 100 & $95^{* *}$ \\
Soft & 0 & 0 & 0 & $5^{* *}$ \\
Diarrhea & 0 & 0 & 0 & 0 \\
\hline
\end{tabular}

**P $<0.01$ versus CPH + DW-treated rat using Steel's test $(n=10)$.

induces cellular movement of sodium and water into the intestinal lumen (Jiang et al., 2015). Furthermore, it promotes the synthesis of intracellular cGMP and subsequently stimulates the cGMP-dependent protein kinase II to phosphorylate CFTR (Vaandrager et al., 1998; Sindic and Schlatter, 2006; Bryant et al., 2010). Additionally, GC-C/cGMP activation plays a role in relieving abdominal pain (Corsetti and Tack, 2013). This new approach improves the difficulty or pain associated with intestinal transit by promoting bowel movements and, therefore, may be beneficial for patients with constipation (Chokhavatia et al., 2016).
The present study demonstrates that MNG enhanced the small intestinal fluid secretion. Moreover, it may be involved in promoting bowel movements by softening feces. Therefore, to clarify the underlying mechanism of action, the effects of MNG on the chloride channels, ClC-2 and CFTR, were examined in vitro. The results revealed that MNG exhibited agonistic activity on CFTR; CFTR channels are distributed in the apical membranes of intestinal epithelial cells (Matthews, 2002; Field, 2003; Yang et al., 2011), and MNG acted locally in the intestinal tract rather than systemically. The concentration of MNG used in vivo was 30 or $60 \mathrm{mg} / \mathrm{mL}$. Although the

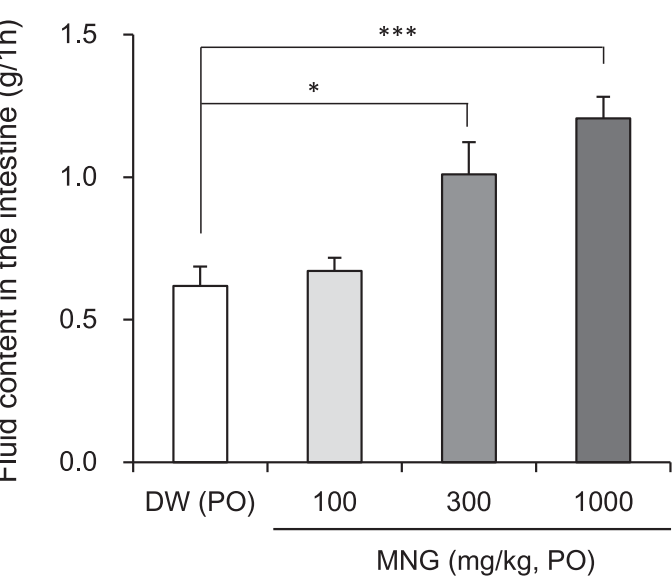

B

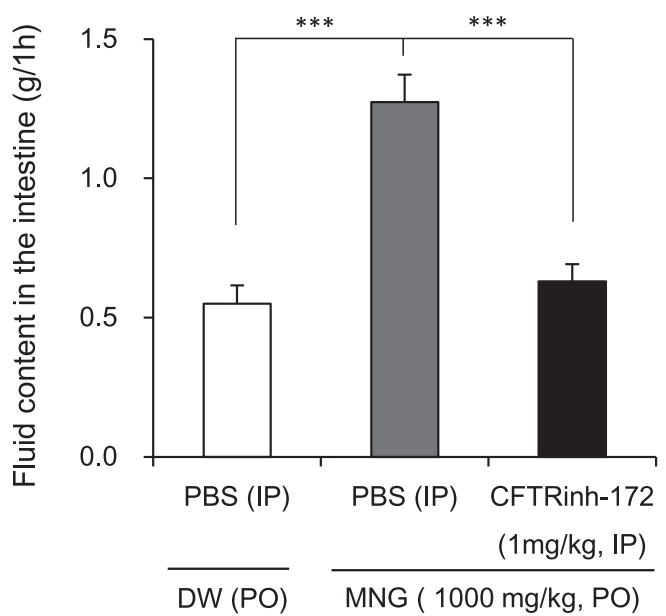

Fig. 4. Effects of MNG on small intestinal fluid secretion. (A) Small intestinal fluid secretion was measured 1 hour after PO administration of DW or MNG in 24-hour fasted normal rats $(* P<0.05$ and $* * * P<$ 0.001 versus DW-treated group by Dunnett's test, $n=4$ ). (B) Vehicle or CFTRinh-172 $(1 \mathrm{mg} / \mathrm{kg})$ was IP injected into rats 0.5 hour before DW or MNG administration $(* * * P<$ 0.001 versus MNG + PBS group by Dunnett's test, $n=10$ ). (C) Vehicle or CFTRinh-172 $1 \mathrm{mg} / \mathrm{kg}$ was IP injected 0.5 hour before DW $(n=6)$. Data are mean \pm S.E.M. Not significant (N.S.) by Student's $t$ test.

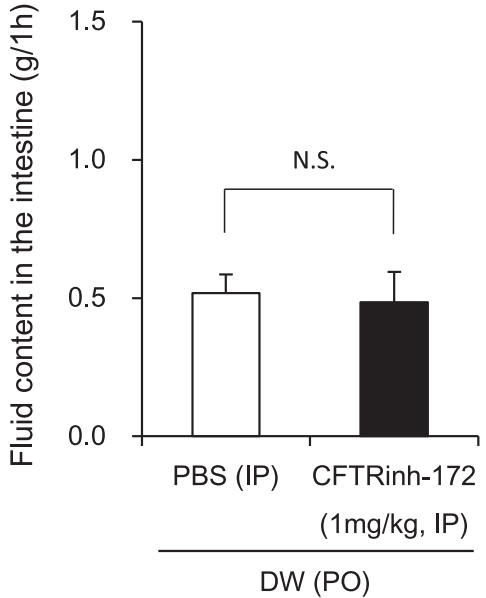


TABLE 3

Effects of MNG on CFTR chloride channel activity evaluated using Ussing chambers assay.

Short-circuit current was changed dose-dependently by treatment with MNG (50-400 $\mu \mathrm{g} / \mathrm{mL})$, and the response was blocked by CFTRinh-172 $(20 \mu \mathrm{mol} / \mathrm{L})$.

\begin{tabular}{lc}
\hline Test Article $(\mu \mathrm{g} / \mathrm{mL})$ & Short-Circuit Current $\left(\mu \mathrm{A} / \mathrm{cm}^{2}\right)$ \\
\hline Vehicle & $0.02 \pm 0.13$ \\
+CFTRinh-172 & $16.81 \pm 1.31$ \\
MNG 50 & $-3.04 \pm 0.26^{* * *}$ \\
MNG 100 & $-5.21 \pm 0.44^{* * *}$ \\
MNG 200 & $-6.73 \pm 0.32^{* * *}$ \\
MNG 400 & $-9.85 \pm 0.17^{* * *}$ \\
+CFTRinh-172 & $23.10 \pm 0.69$ \\
\hline
\end{tabular}

***P $<0.01$ versus vehicle by Dunnett's test. Data are mean \pm S.E.M. $(n=3)$.

strict concentration of MNG delivered to the small intestinal lumen is unknown, we considered that MNG could reach the levels (50-400 $\mu \mathrm{g} / \mathrm{mL})$ used in the Ussing chamber experiments. Furthermore, the increase in the small intestinal fluid content induced by MNG treatment was completely abolished by coadministration with CFTRinh-172. These results suggest that MNG enhanced secretion of the small intestinal fluid by activating CFTR chloride channels. Conversely, MNG did not activate ClC-2 channel. Recent studies demonstrated that ClC-2 is mostly located in proximity to tight junctions, intercellular membranes, and basolateral membranes, and its distribution varies from species to species (Jin and Blikslager, 2015). It suggests that the ClC-2 chloride channel has a critical role in the regulation of intestinal barrier function, colonic $\mathrm{NaCl}$ absorption, and cell volume, rather than secretion.

MNG contains multiple components, but the active ingredient mediating its efficacy was not identified in this study. Some studies have demonstrated the pharmacological effects of crude drugs contained in MNG. Cannabis Fructus contains a large amount of fatty acid oils, including olein, linolein, and linolenin, which affect the small intestinal fluid secretion (Slota et al., 1983). Naringenin, an ingredient of Aurantii Fructus Immaturus, increased the production of cGMP by inhibiting phosphodiesterase activity (Orallo et al., 2005). These findings suggest the activation of CFTR chloride channel through cGMP signal transduction. In contrast, Rhei Rhizoma-containing drugs are widely used as laxatives (Iizuka and Hamamoto, 2015; Hirose et al., 2016). MNG also contains Rhei Rhizoma; however, it improved constipation without inducing diarrhea in rats in this study. In addition, the Kampo medicine, daioukanzoto, which is composed of Rhei Rhizoma and Glycyrrhizae radix, did not have CFTR activity (data not shown). This observation suggested that Rhei Rhizoma was not likely to be involved in the increase in small intestinal fluids induced by MNG. However, Rhei Rhizoma enhances intestinal movement (Iizuka and Hamamoto, 2015; Hirose et al., 2016). It is well known that sennosides A and B, which are present in Rhei Rhizoma, are metabolized by intestinal bacteria to become rheinanthrone, which has strong laxative activity. Increased myoelectric activity and contractility in the intestine and colon by Rhizoma-containing drugs suggest that this may contribute to the reversal of constipation by promoting motility and accelerating transit. Magnolol and honokiol, which are constituents of Magnoliae Cortex, promote gastrointestinal motility (Zhang et al., 2005; Yang et al., 2008; Miao et al., 2013). Peoniflorin, a constituent of
Paeoniae Radix, is known to relieve pain and may have a beneficial effect on abdominal pain in patients with constipation (Zhang et al., 2008, 2009; Xu et al., 2013). These beneficial effects that improve symptoms of patients with constipation are also likely to be synergistically involved in the efficacy of MNG. However, we did not identify the active ingredient of MNG and its specific detailed mechanism of action for correlating opioid receptors and CFTR; therefore, further investigation is required.

In the present study, we used a rat model in which constipation was induced by codeine, a relatively weak opioid agonist. It has been reported that the CFTR activator, phenylquinoxalinone, normalized stool output and water content in a mouse model of constipation induced by the more potent opioid agonist loperamide (Cil et al., 2016). These findings suggested that CFTR-mediated stool softening overcame opioid-induced constipation. Moreover, a clinical study of MNG on functional constipation symptoms demonstrated that most patients tolerated MNG well and no serious adverse effect was found. However, further investigation is needed to assess the safety of MNG, particularly in the elderly, who often have multiple comorbidities, and patients undergoing chemotherapy.

In summary, this study provided evidence to support an undoubtedly major role for MNG in improving constipation by, at least in part, increasing the intestinal fluid secretion through CFTR chloride channel activation.

In conclusion, we demonstrated that MNG increased the fecal output in opioid-induced constipation in rats. Furthermore, our results suggested that the effects of MNG are mediated by increasing the small intestinal fluid secretion by CFTR chloride channel activation. Therefore, MNG could be used for the treatment of constipation in patients taking opioids.

\section{Acknowledgments}

We thank Dr. A Nakajima for helpful discussion.

\section{Authorship Contributions}

Participated in research design: Harada, Fujitsuka.

Conducted experiments: Harada, Iizuka, Saegusa, Mogami.

Performed data analysis: Harada, Saegusa.

Wrote or contributed to the writing of the manuscript: Harada, Fujitsuka, Hattori.

\section{References}

Akiba Y, Jung M, Ouk S, and Kaunitz JD (2005) A novel small molecule CFTR inhibitor attenuates HCO3- secretion and duodenal ulcer formation in rats. Am J Physiol Gastrointest Liver Physiol 289:G753-G759.

Bhol KC and Schechter PJ (2007) Effects of nanocrystalline silver (NPI 32101) in a rat model of ulcerative colitis. Dig Dis Sci 52:2732-2742.

Bijvelds MJ, Bot AG, Escher JC, and De Jonge HR (2009) Activation of intestinal Clsecretion by lubiprostone requires the cystic fibrosis transmembrane conductance regulator. Gastroenterology 137:976-985.

Bryant AP, Busby RW, Bartolini WP, Cordero EA, Hannig G, Kessler MM, Pierce CM, Solinga RM, Tobin JV, Mahajan-Miklos S, et al. (2010) Linaclotide is a potent and selective guanylate cyclase $\mathrm{C}$ agonist that elicits pharmacological effects locally in the gastrointestinal tract. Life Sci 86:760-765.

Busby RW, Bryant AP, Bartolini WP, Cordero EA, Hannig G, Kessler MM, MahajanMiklos S, Pierce CM, Solinga RM, Sun LJ, et al. (2010) Linaclotide, through activation of guanylate cyclase $\mathrm{C}$, acts locally in the gastrointestinal tract to elicit enhanced intestinal secretion and transit. Eur J Pharmacol 649:328-335.

Camilleri M, Bharucha AE, Ueno R, Burton D, Thomforde GM, Baxter K, McKinzie $\mathrm{S}$, and Zinsmeister AR (2006) Effect of a selective chloride channel activator, lubiprostone, on gastrointestinal transit, gastric sensory, and motor functions in healthy volunteers. Am J Physiol Gastrointest Liver Physiol 290:G942-G947.

Canadian Agency for Drugs and Technologies in Health (2014) Treatments for Constipation: A Review of Systematic Reviews. [Internet] Ottawa, Ontario, Canada.

Cheng CW, Bian ZX, Zhu LX, Wu JC, and Sung JJ (2011) Efficacy of a Chinese herbal proprietary medicine (hemp seed pill) for functional constipation. Am J Gastroenterol 106:120-129. 
Chokhavatia S, John ES, Bridgeman MB, and Dixit D (2016) Constipation in elderly patients with noncancer pain: focus on opioid-induced constipation. Drugs Aging 33:557-574.

Cil O, Phuan PW, Lee S, Tan J, Haggie PM, Levin MH, Sun L, Thiagarajah JR, Ma T, and Verkman AS (2016) CFTR activator increases intestinal fluid secretion and normalizes stool output in a mouse model of constipation. Cell Mol Gastroenterol Hepatol 2:317-327.

Cil O, Phuan PW, Son JH, Zhu JS, Ku CK, Tabib NA, Teuthorn AP, Ferrera L, Zachos NC, Lin R, et al. (2017) Phenylquinoxalinone CFTR activator as potential prosecretory therapy for constipation. Transl Res. 182:14-26.e4.

Corsetti M and Tack J (2013) Linaclotide: a new drug for the treatment of chronic constipation and irritable bowel syndrome with constipation. United European Gastroenterol J 1:7-20.

Costilla VC and Foxx-Orenstein AE (2014) Constipation in adults: diagnosis and management. Curr Treat Options Gastroenterol 12:310-321.

De Lisle RC (2012) Lubiprostone stimulates small intestinal mucin release. BMC Gastroenterol 12:156.

Field M (2003) Intestinal ion transport and the pathophysiology of diarrhea. J Clin Invest 111:931-943.

Fredericks A, Hollis G, and Stricker CT (2010) Diagnosis and management of opioidinduced bowel dysfunction in patients with advanced cancer. Clin J Oncol Nurs 14 701-704.

Gras-Miralles B and Cremonini F (2013) A critical appraisal of lubiprostone in the treatment of chronic constipation in the elderly. Clin Interv Aging 8:191-200.

Gyawali B, Hayashi N, Tsukuura H, Honda K, Shimokata T, and Ando Y (2015) Opioid-induced constipation. Scand J Gastroenterol 50:1331-1338.

Harada Y, Iizuka S, Saegusa Y, Mogami S, Fujitsuka N, Hattori T, Kase Y, and Nakajima A (2016) Effect of mashiningan on fiber-free diet-induced defecation disorder in rats. Jpn Pharmacol Ther 44:1435-1443.

Hirose T, Shinoda Y, Yoshida A, Kurimoto M, Mori K, Kawachi Y, Tanaka K, Takeda A, Yoshimura T, and Sugiyama T (2016) Efficacy of daiokanzoto in chronic constipation refractory to first-line laxatives. Biomed Rep 5:497-500.

Iizuka N and Hamamoto Y (2015) Constipation and herbal medicine. Front Pharmacol 6:73.

Izzy M, Malieckal A, Little E, and Anand S (2016) Review of efficacy and safety of laxatives use in geriatrics. World J Gastrointest Pharmacol Ther 7:334-342.

Jakab RL, Collaco AM, and Ameen NA (2012) Lubiprostone targets prostanoid signaling and promotes ion transporter trafficking, mucus exocytosis, and contractility. Dig Dis Sci 57:2826-2845.

Jiang C, Xu Q, Wen X, and Sun H (2015) Current developments in pharmacological therapeutics for chronic constipation. Acta Pharm Sin B 5:300-309.

Jin Y and Blikslager AT (2015) ClC-2 regulation of intestinal barrier function: translation of basic science to therapeutic target. Tissue Barriers 3:e1105906.

Kurniawan I and Simadibrata M (2011) Management of chronic constipation in the elderly. Acta Med Indones 43:195-205.

Lacy BE and Levy LC (2007) Lubiprostone: a chloride channel activator. J Clin Gastroenterol 41:345-351.

LoCasale RJ, Datto C, Margolis MK, and Coyne KS (2016) Satisfaction with therapy among patients with chronic noncancer pain with opioid-induced constipation. $J$ Manag Care Spec Pharm 22:246-253.

Matthews JB (2002) Molecular regulation of Na+-K+-2Cl- cotransporter (NKCC1) and epithelial chloride secretion. World J Surg 26:826-830.

McNicol E, Horowicz-Mehler N, Fisk RA, Bennett K, Gialeli-Goudas M, Chew PW, Lau J, and Carr D (2003) Management of opioid side effects in cancer-related and chronic noncancer pain: a systematic review. J Pain 4:231-256.

Miao B, Zhang S, Wang H, Yang T, Zhou D, and Wang BE (2013) Magnolol pretreatment prevents sepsis-induced intestinal dysmotility by maintaining functional interstitial cells of Cajal. Inflammation 36:897-906.

Mizumori M, Akiba Y, and Kaunitz JD (2009) Lubiprostone stimulates duodenal bicarbonate secretion in rats. Dig Dis Sci 54:2063-2069.

Nakae Y, Kosuge T, Kumagai Y, and Tanaka F (2016) The effectiveness of mashiningan for constipation in Parkinson's disease. Kampo Med 67:131-136.
Nelson AD and Camilleri M (2016) Opioid-induced constipation: advances and clinical guidance. Ther Adv Chronic Dis 7:121-134.

Orallo F, Camiña M, Alvarez E, Basaran H, and Lugnier C (2005) Implication of cyclic nucleotide phosphodiesterase inhibition in the vasorelaxant activity of the citrus-fruits flavonoid (+/-)-naringenin. Planta Med 71:99-107.

Prichard D, Norton C, and Bharucha AE (2016) Management of opioid-induced constipation. Br J Nurs 25:S4-S5, S8-S11.

Sani H and Mahan RJ (2015) Treating opioid-induced constipation in older adults: new options. Consult Pharm 30:612-615.

Schiller LR (2004) New and emerging treatment options for chronic constipation. Rev Gastroenterol Disord 4 (Suppl 2):S43-S51.

Sharma S, Sharma T, Dhingra R, Tomar P, Singh S, Malhotra M, and Bhardwaj TR (2013) Linaclotide: a novel secretagogue in the treatment of irritable bowel syndrome with constipation and chronic idiopathic constipation. Mini Rev Med Chem 13:1685-1690.

Sindić A and Schlatter E (2006) Cellular effects of guanylin and uroguanylin. J Am Soc Nephrol 17:607-616.

Slota T, Kozlov NA, and Ammon HV (1983) Comparison of cholesterol and betasitosterol: effects on jejunal fluid secretion induced by oleate, and absorption from mixed micellar solutions. Gut 24:653-658.

Thiagarajah JR, Broadbent T, Hsieh E, and Verkman AS (2004) Prevention of toxininduced intestinal ion and fluid secretion by a small-molecule CFTR inhibitor. Gastroenterology 126:511-519.

Vaandrager AB, Smolenski A, Tilly BC, Houtsmuller AB, Ehlert EM, Bot AG, Edixhoven M, Boomaars WE, Lohmann SM, and de Jonge HR (1998) Membrane targeting of cGMP-dependent protein kinase is required for cystic fibrosis transmembrane conductance regulator Cl- channel activation. Proc Natl Acad Sci USA 95:1466-1471.

Xu CH, Wang P, Wang Y, Yang Y, Li DH, Li HF, Sun SQ, and Wu XZ (2013) Pharmacokinetic comparisons of two different combinations of Shaoyao-Gancao Decoction in rats: competing mechanisms between paeoniflorin and glycyrrhetinic acid. J Ethnopharmacol 149:443-452.

Yang H, Xu LN, He CY, Liu X, Fang RY, and Ma TH (2011) CFTR chloride channel as a molecular target of anthraquinone compounds in herbal laxatives. Acta Pharmacol Sin 32:834-839.

Yang TC, Zhang SW, Sun LN, Wang H, and Ren AM (2008) Magnolol attenuates sepsis-induced gastrointestinal dysmotility in rats by modulating inflammatory mediators. World $J$ Gastroenterol 14:7353-7360.

Yu SW and Rao SS (2014) Advances in the management of constipation-predominant irritable bowel syndrome: the role of linaclotide. Therap Adv Gastroenterol 7: 193-205.

Zhang WW, Li Y, Wang XQ, Tian F, Cao H, Wang MW, and Sun QS (2005) Effects of magnolol and honokiol derived from traditional Chinese herbal remedies on gastrointestinal movement. World J Gastroenterol 11:4414-4418.

Zhang XJ, Chen HL, Li Z, Zhang HQ, Xu HX, Sung JJ, and Bian ZX (2009) Analgesic effect of paeoniflorin in rats with neonatal maternal separation-induced visceral hyperalgesia is mediated through adenosine $\mathrm{A}(1)$ receptor by inhibiting the extracellular signal-regulated protein kinase (ERK) pathway. Pharmacol Biochem Behav 94:88-97.

Zhang XJ, Li Z, Leung WM, Liu L, Xu HX, and Bian ZX (2008) The analgesic effect of paeoniflorin on neonatal maternal separation-induced visceral hyperalgesia in rats. J Pain 9:497-505.

Zhong LL, Cheng CW, Chan Y, Chan KH, Lam TW, Chen XR, Wong CT, Wu JC, and Bian ZX (2013) Chinese herbal medicine (Ma Zi Ren Wan) for functional constipation: study protocol for a prospective, double-blinded, double-dummy, randomized controlled trial. Trials 14:366.

Address correspondence to: Dr. Naoki Fujitsuka, Tsumura Kampo Research Laboratories, Tsumura, 3586 Yoshiwara, Ami-machi, Inashiki-gun, Ibaraki 300-1192, Japan. E-mail: fujitsuka_naoki@mail.tsumura.co.jp 